\title{
Dynamic Eco-Driving Speed Guidance at Signalized Intersections: Multivehicle Driving Simulator Based Experimental Study
}

\author{
Peng Chen $(D),{ }^{1,2}$ Cong Yan, ${ }^{1}$ Jian Sun $(D), 3$ Yunpeng Wang, \\ Shenyang Chen, ${ }^{4}$ and Keping $\mathrm{Li}^{3}$ \\ ${ }^{1}$ Beijing Key Laboratory for Cooperative Vehicle Infrastructure Systems and Safety Control, School of \\ Transportation Science and Engineering, Beihang University, Xue Yuan Road No. 37, Hai Dian District, Beijing 100191, China \\ ${ }^{2}$ Beijing Advanced Innovation Center for Big Data and Brain Computing, Beihang University, Xue Yuan Road No. 37, \\ Hai Dian District, Beijing 100191, China \\ ${ }^{3}$ Department of Traffic Engineering and Key Laboratory of Road and Traffic Engineering, Ministry of Education, \\ Tongji University, Shanghai 201804, China \\ ${ }^{4}$ Department of Civil and Environmental Engineering, University of California, Davis, CA 95616, USA
}

Correspondence should be addressed to Jian Sun; sunjian@tongji.edu.cn

Received 12 July 2017; Revised 12 December 2017; Accepted 21 December 2017; Published 7 May 2018

Academic Editor: Antonino Vitetta

Copyright (C) 2018 Peng Chen et al. This is an open access article distributed under the Creative Commons Attribution License, which permits unrestricted use, distribution, and reproduction in any medium, provided the original work is properly cited.

\begin{abstract}
Variations in vehicle fuel consumption and gas emissions are usually associated with changes in cruise speed and the aggressiveness of drivers' acceleration/deceleration, especially at traffic signals. In an attempt to enhance vehicle fuel efficiency on arterials, this study developed a dynamic eco-driving speed guidance strategy (DESGS) using real-time signal timing and vehicle positioning information in a connected vehicle (CV) environment. DESGS mainly aims to optimize the fuel/emission speed profiles for vehicles approaching signalized intersections. An optimization-based rolling horizon and a dynamic programming approach were proposed to track the optimal guided velocity for individual vehicles along the travel segment. In addition, a vehicle specific power (VSP) based approach was integrated into DESGS to estimate the fuel consumption and $\mathrm{CO}_{2}$ emissions. To evaluate the effectiveness of the overall strategy, 15 experienced drivers were recruited to participate in interactive speed guidance experiments using multivehicle driving simulators. It was found that compared to vehicles without speed guidance, those with DESGS had a significantly reduced number of stops and approximately $25 \%$ less fuel consumption and $\mathrm{CO}_{2}$ emissions.
\end{abstract}

\section{Introduction}

The transportation sector not only accounts for a vast quantity of fuel consumption but also serves as the largest atmospheric carbon emitter in the world. Concerns over energy security, environmental protection, and economic benefits have prompted renewed public and private interest in an ecological energy-efficient driving style that can reduce fuel consumption and greenhouse gas emissions. Eco-driving serves as one effective and cost-effective strategy to enhance the fuel efficiency of the transportation section [1-3]. The essential idea of eco-driving is to provide real-time driving advice to individual vehicles so that the drivers can adjust their driving behavior or take driving actions to reduce fuel consumption and emission levels [4].

The Intelligent Speed Adaptation (ISA) initiative in the UK aimed to develop driver assistive devices that recommend desired speeds to avoid hard braking [5]. Even though the primary objective of an ISA device is safer driving, its users benefitted from reduced fuel consumption and emissions due to smoother speed variations [6]. From an energy and emissions standpoint, this initiative laid the foundation for eco-driving, which refers to driving in an ecological and economical way.

The effects of driving behavior on fuel consumption and emissions have been widely studied [7-9]. Yang et al. [10] 
summarized that frequent acceleration associated with stopand-go waves, excessive speeds, slow movement on congested roads, and extra idling times are major causes of increased fuel consumption and emissions. It is well recognized that maintaining a constant velocity and avoiding unnecessary acceleration and deceleration are the key principles of ecodriving [11].

To develop eco-driving strategies on freeways appears straightforward considering that the traffic flow is continuous and vehicles are seldom interrupted by traffic control devices [12]. The eco-driving strategy can simply compute advisory speeds or speed limits for drivers. Unlike freeways, the traffic flows on arterials are essentially interrupted because of complex interactions between volatile traffic patterns and signal control as well as the interdependent relationships of vehicle queues at neighboring intersections under different levels of congestion [13]. The dependency of speed variations on external factors, such as neighboring vehicles, signal status, and other infrastructural limitations, results in yet limited use of eco-driving on arterials.

Numerical empirical studies show a positive relationship between vehicle emissions and fuel consumption with delays at traffic signals $[14,15]$. According to the state of practice, signal coordination ranks as one of the most effective control strategies for solving energy and fuel consumption problems on arterials. The core concept of this strategy is to coordinate a number of traffic signals by adjusting the offset between neighboring intersections to optimize travel speeds, reduce delays, and minimize stops. However, constraints in field operation, such as oversized spacing between adjacent intersections, complicated traffic composition, and the diversity of driving behavior, may lead to decreased reliability and stability of the coordinated signal control system, which is characterized by a discrete form of traffic or a large difference between running speed and preset speed [16, 17]. In such cases, signal coordination fails to reach its design goals and may result in increased fuel consumption, trip time, and engine and brake wear.

Recently, the emerging connected vehicle (CV) technology initiatively led by the U.S. Department of Transportation has encouraged increased planning and implementation [18]. Vehicle-to-Vehicle (V2V) and Vehicle-to-Infrastructure (V2I) communications offer the potential to obtain real-time vehicle positions and traffic signal timing at downstream locations. Speed can be adjusted for timely arrival at green lights, which enables an eco-friendly driving style. Thus, improving the traffic environment and decreasing emissions with arterial eco-driving strategies have become an achievable goal [19]. There has been increasing efforts to develop eco-driving strategies for assisting drivers to travel along signalized arterials.

Mandava et al. [20] developed an arterial velocity planning algorithm that provides dynamic speed advice to the driver to maximize the probability of having a green light when approaching signalized intersections. The algorithm aimed to minimize the acceleration/deceleration rates within the speed limit while avoiding complete stops. Last, it was tested by using a stochastic simulation technique along a 10intersection signalized corridor. However, it was argued that lowering acceleration/deceleration levels does not necessarily indicate reducing fuel consumption. Asadi and Vahidi [21] used upcoming traffic signal information within the vehicle's adaptive cruise control system to reduce idle time at stop lights and fuel consumption. The control objectives were set as timely arrival at green light with minimal use of braking, maintaining safe distance between vehicles, and cruising at or near set speed. Barth et al. [22] developed a dynamic eco-driving velocity planning algorithm to minimize acceleration/deceleration rates, the total tractive power demand, and the idling time for probe vehicles, so that fuel consumption and emissions can be reduced. Rakha and Kamalanathsharma [23] presented seven examples with different speeds and throttles through a sample scenario with a single signalized intersection. After assuming or calculating the other parameters, they determined which case had the most fuel-optimal speed profile. Recently, Yang et al. [10] developed an Eco-Cooperative Adaptive Cruise Control (Eco-CACC) algorithm to compute the fueloptimum vehicle trajectory through a signalized intersection by ensuring that the vehicle arrives at the intersection stop bar just as the last queued vehicle is discharged. The key of the algorithm is to predict the queue length ahead of the probe vehicles using the LWR and the release time of the queue using the signal phasing and timing (SPaT) data. Last, the algorithm was tested using the INTEGRATION microscopic traffic simulation software. It was found that the queue prediction enhanced the Eco-CACC algorithm to produce the smoothest vehicle trajectories and to result in the highest fuel savings, approximately $11.4 \%$.

In the aforementioned studies, the goal of reducing fuel consumption was usually transformed into simple functions of acceleration/deceleration rates [20,22] or the time of arrival at the intersection $[10,21,23]$ (Sun and Henry, 2015; Wu et al, 2015a, 2015b). None of these models used an explicit objective function to minimize the total fuel consumption. In addition, most of these systems do not provide predictive ecodriving control strategies that optimize the vehicle controls in advance. Moreover, their findings were obtained through theoretical calculations or microscopic simulation instead of experimental validation or field testing. Last but not least, even for the experimental studies, they failed to consider driver responses and the interactions between human drivers and traffic flow.

Thus, this study developed a dynamic eco-driving speed guidance strategy (DESGS) to help optimize the fuel/emission speed profiles for vehicles approaching signalized intersections. Based on the signal timing and vehicle position information, an optimization algorithm was proposed for drivers to achieve minimum fuel consumption and $\mathrm{CO}_{2}$ emissions. In addition, a vehicle specific power (VSP) based approach was integrated into DESGS to estimate the fuel consumption and $\mathrm{CO}_{2}$ emissions. Furthermore, the proposed strategy was investigated on a typical arterial in Shanghai, China, using multivehicle driving simulators, which would enable us to integrate the real driver responses. As an initial step, the developed DESGS helps highlight the importance of retaining microscopic fuel consumption models in the optimization function and in the predictive 


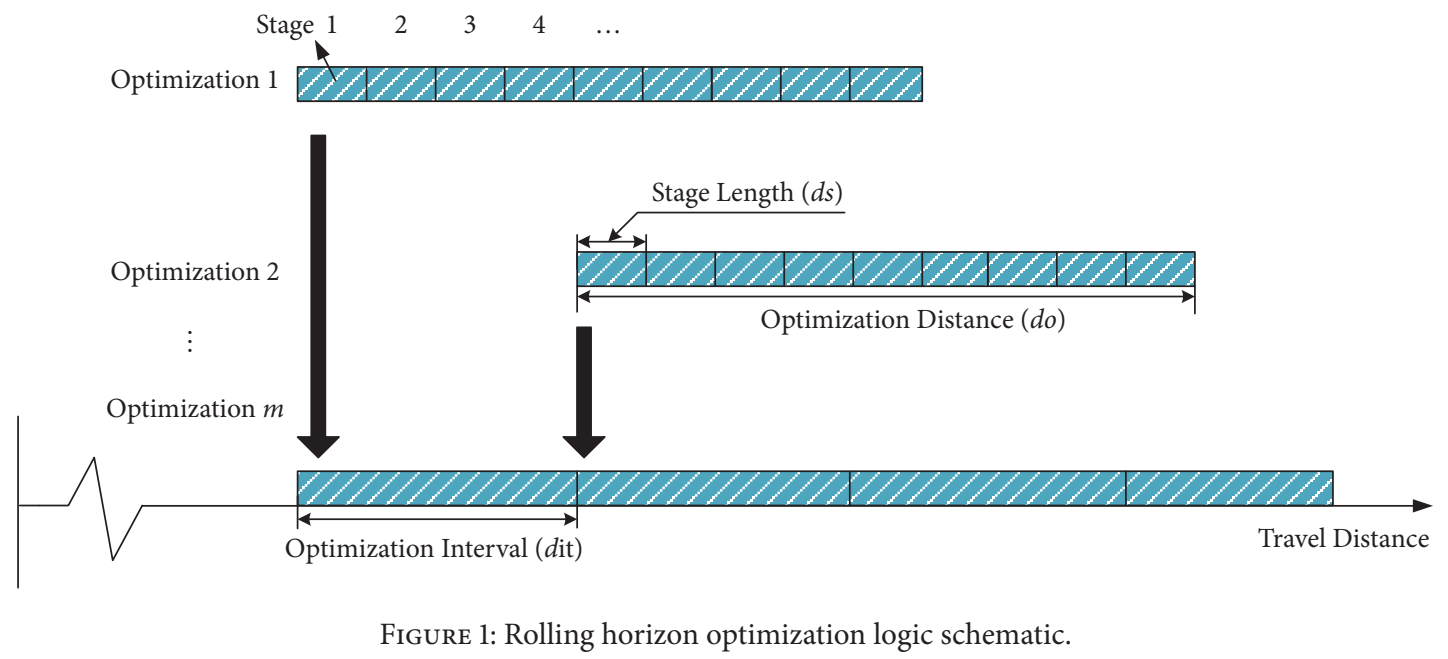

model to minimize fuel consumption and $\mathrm{CO}_{2}$ emissions for a given road topography.

The paper is organized as follows. Section 2 presents the methodology of DESGS for eco-driving speed calculations. Section 3 presents a case study based on multivehicle driving simulators for a signalized arterial. Section 4 analyzes the benefits and efficiency of the proposed DESGS. Section 5 offers concluding remarks and directions for future work.

\section{Methodology}

The DESGS consists of two building blocks, that is, an optimization module and an eco-index calculation module. The guided velocity is dynamically adjusted based on the vehicle's spatial-temporal trajectories using an optimizationbased rolling horizon and a dynamic programming (DP) approach. Given the topological information and driving characteristics of the test vehicles, the eco-index calculation module can estimate the fuel consumption rates based on the vehicle operating conditions. The optimization module is then used to find an optimal control plan that minimizes the total fuel consumption and $\mathrm{CO}_{2}$ emissions, while satisfying the preset minimum and maximum vehicle speeds.

\subsection{Optimization Module}

2.1.1. Rolling Horizon Optimization Logic. The optimization module is the most important component that defines the structure of the system. A rolling horizon approach is used for optimization based on the most recent predictions and observations. As illustrated in Figure 1, this module consists of three parameters: (i) the stage length $\left(d_{s}\right)$ is the unit of discretization; (ii) the optimization distance $\left(d_{o}\right)$, which is also referred to as the look-ahead distance, is the unit of optimization; and (iii) the optimization interval $\left(d_{\mathrm{it}}\right)$ represents the optimization frequency.

In this study, a guided speed decision is made for the optimization distance $\left(d_{o}\right)$ at the position where a vehicle starts to receive speed advice. Here, $d_{o}$ indicates the distance from the current position to the estimated position where the vehicle will pass through the intersection. To calculate the stage length $\left(d_{s}\right)$ at the beginning of stage $i$, this study assumes that a vehicle begins to accelerate or decelerate at a feasible rate from an initial velocity to a guided velocity and then cruises at this velocity over this distance. Considering the minimum and maximum values of the highway speed limit, the engine force, and the braking torque ability, $d_{s}$ is typically $100-200 \mathrm{~m}$. In the optimization interval $\left(d_{\mathrm{it}}\right)$, a vehicle cruises at the guided velocity over stage $i(i=2,3,4, \ldots)$ after traveling through $d_{s}$ of stage 1 until reaching the end of $d_{\mathrm{it}}$. Since the guided velocity may not agree with the vehicle's current velocity, $d_{\text {it }}$ should be sufficiently long to avoid the driver frequently changing vehicle speed or causing driver boredom. On the other hand, when $d_{\text {it }}$ is too long, the chance of the vehicle hitting a red light increases, corresponding to a required speed change and a guidance failure. Thus, $d_{\mathrm{it}}$ was assigned a value of $400 \mathrm{~m}$ in this study. A new decision is made with updated parameters at the end of $d_{\text {it }}$.

2.1.2. Dynamic Programming (DP) implementation. There are three basic approaches for addressing optimal control problems, that is, DP, direct methods [24, 25] and indirect methods [26, 27]. DP adopts the principle of optimality of subarcs to recursively compute feedback control. In the continuous case, this method leads to the Hamilton-JacobiBellman equation. DP can easily handle nonconvex control problems and inequality constraints [28]. Thus, this study uses DP to solve the minimum fuel vehicle driving problem.

At the beginning of each optimization interval $\left(d_{\mathrm{it}}\right)$, the optimization module finds an optimal control plan using Dijkstra's shortest path algorithm, which was developed specifically for this application in light of the cost function matrix.

As shown in Figure 2, the variables in the solid circle represent all the possible guided velocities $\left(v_{g}\right)$ that may be displayed by each guided position. The value of $v_{g}$ is given by

$$
\begin{aligned}
v_{g} & =5 \cdot n, \quad n=1,2,3, \ldots \\
v_{\min } & \leq v_{g} \leq v_{\max },
\end{aligned}
$$




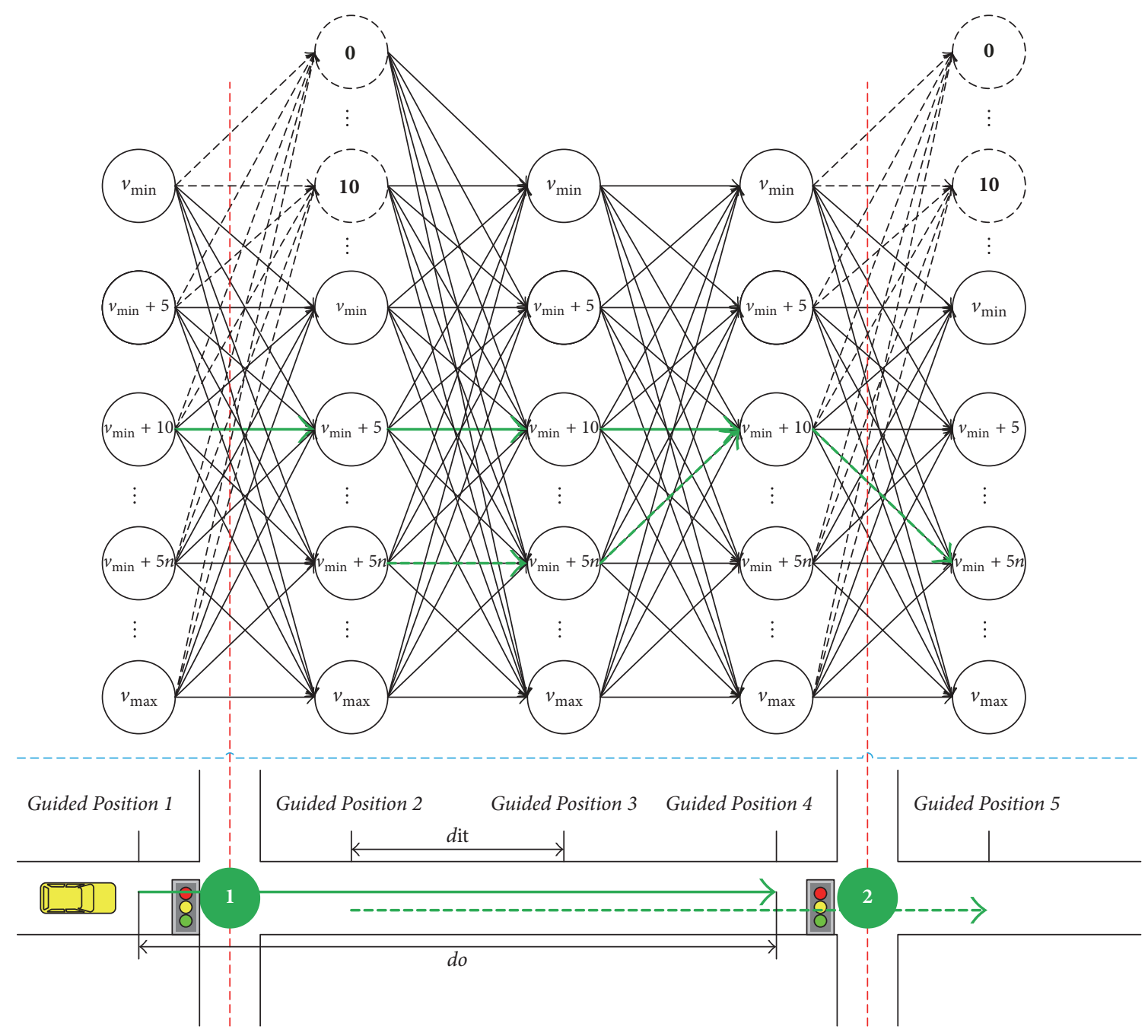

FIGURE 2: The DESGS calculation using DP.

where $v_{g}$ is set as multiples of $5 \mathrm{~km} / \mathrm{h}$ to facilitate the DESGS calculation process; $v_{\min }$ is the minimum speed limit of the study link, which is set to protect the speed guidance strategy from impairing traffic mobility or safety; $v_{\max }$ is the maximum speed limit, which is taken as the smaller value between the maximum speed limit of the link and the highest vehicle speed based on power performance. The numbers in the dotted circle represent the possible actual velocities when the vehicle crosses the intersection stop line because of the obstruction of a red light. Note that $v_{k}^{\prime}$ is the velocity of the vehicle when it arrives at the intersection stop line. The vehicle travels at a specific $v_{g}$ from the last guided position to the downstream intersection stop line. In this process, when the signal light is always green, $v_{k}^{\prime}=v_{g}$; when the driver observes that the light is red and starts to decelerate but the light turns green as the vehicle reaches the stop line, $0<v_{k}^{\prime} \leq$ $v_{g}$; when the signal is always red, $v_{k}^{\prime}=0$.

The distance between the adjacent guided positions is defined as one optimization interval $\left(d_{\text {it }}\right)$. For example, as a vehicle reaches guided position 1 , the optimization module will calculate the fuel consumption along the travel segment considering all the possible combinations of guided velocity over one optimization distance $\left(d_{o}\right)$. This distance is based on the signal timing parameters at the next downstream intersection, the queue length at the approach, and/or the leading vehicles. In the computation process, the influence of the signal lights between adjacent guided positions, which may lead to the vehicle traveling on the segment, does not consider the recommended velocity at former positions. For example, the guided velocity displayed at position 1 is $70 \mathrm{~km} / \mathrm{h}$, but the vehicle traveling at this speed to the downstream intersection will meet a red light. In this case, the vehicle will immediately brake and decelerate to the stop line. After the vehicle clears the intersection, its task is to accelerate back to the initial velocity $(70 \mathrm{~km} / \mathrm{h})$, assuming that drivers completely conform to the guided velocity. This process will increase redundant fuel consumption and emissions due to the accelerations and decelerations required at the signals. Thus, we can easily infer that this guidance effect is not ideal. When a vehicle travels the same distance, for example, from guided position 1 to position 2 and from guided position 2 to position 3, with the same speed change value, for example, 
from $70 \mathrm{~km} / \mathrm{h}$ to $60 \mathrm{~km} / \mathrm{h}$, the fuel consumption may be different because the vehicle may meet a red light in one of the segments, resulting in excess fuel consumption.

After comparing the total fuel consumption of each combination, the "guided velocity ribbon" will be determined for the smallest fuel consumption, as illustrated by the solid green line in Figure 2. That is, the actual guided velocity issued by guided position 1 is $\left(v_{\min }+10\right) \mathrm{km} / \mathrm{h}$. Similarly, as the vehicle reaches guided position 2 , the green dotted line is obtained using the same algorithm. The speed advice provided by the guided position is in real-time based on the vehicle's current velocity, the signal phase and timing information, and the distance to the next downstream intersection. If the driver does not follow the guidance, he/she will receive new speed advice at the next guided position. However, if the driver fully complies with the "guided velocity ribbon," the actual guided velocity at the overlap between two adjacent optimization distance $\left(d_{o}\right)$ should be consistent.

2.2. Objective Function and Constraints. When a vehicle receives speed advice, its initial speed may differ from the guided speed. Additionally, the current state of the signal could be either red or green. In either case, the vehicle must adjust its speed according to the system state at the beginning of each stage 1 .

In this regard, the DESGS aims to provide an explicit objective function to minimize the total fuel consumption when vehicles pass several intersections. The control performance index at each step for the $k$ th vehicle is evaluated in terms of a cost function $L$ to calculate the fuel consumption rates.

$$
\begin{aligned}
L= & w_{1} * \mathrm{EF}_{\left(v_{k}, v_{k}^{\prime \prime}\right)}+w_{2} *\left|v_{k}-v_{g}\right| * \mathrm{EF}_{\left(v_{g}\right)}+w_{3} \\
& * F_{\text {brake }}^{k} *\left(\mathrm{EF}_{\left(v_{k}, v_{k}^{\prime}\right)}+\mathrm{EF}_{\left(v_{k}^{\prime}, v_{k}\right)}\right),
\end{aligned}
$$

where $w_{1}$ is the weight factor for the fuel consumption, $w_{2}$ is the weight factor for the deviation from the target speed, $w_{3}$ is the weight factor for the braking effort, and they are all set as one-third in this study for simplicity; $v_{k}$ is the initial velocity of the $k$ th vehicle, $v_{k}^{\prime \prime}$ is its final velocity, $v_{g}$ is the guided velocity, and $v_{k}^{\prime}$ is the velocity when the $k$ th vehicle crosses the intersection stop line; $\mathrm{EF}_{\left(v_{g}\right)}$ is the fuel consumption at $v_{g}$ over one optimization interval $\left(d_{\mathrm{it}}\right), \mathrm{EF}_{\left(v_{k}, v_{k}^{\prime \prime}\right)}$ is the fuel consumption from $v_{k}$ to $v_{k}^{\prime \prime}$ over one $d_{\mathrm{it}}$, and $F_{\text {brake }}^{k}$ is the braking force of the $k$ th vehicle. The indexes $w_{1}$ and $w_{2}$ penalize the deviations of vehicle speed $v_{k}$ from the guided velocity $v_{g}$, and $w_{3}$ indicates a reduced use of brake force.

To achieve DESGS, we need to add certain constraints for controlling the movement of vehicles. In this study, the speed limit, acceleration and deceleration limits, and the minimum safe following distance are imposed as pointwise-in-time inequality constraints [21]. These constraints are effective over the future prediction horizon.

(1) Speed Limit Constraint. The speed limit constraint is as follows:

$$
v_{\min } \leq v_{k} \leq v_{\max }
$$

As mentioned above, to reduce the fuel consumption and emissions, sharp accelerations and decelerations and idling time should be avoided as much as possible. Note that $t_{t}$ is the total time taken for the vehicle to reach the next signalized intersection after receiving speed advice, and $t_{s}$ is the time until then next signal state changed where $s \in$ \{green, red\}. For example, $t_{\text {green }}$ denotes the time when the signal turns green from the instant when the vehicle receives speed advice. $t_{\text {green }}$ and $t_{\text {red }}$ have number values. Suppose that $t_{\text {green }}(i)$ is the start of the $i$ th green signal state and that $t_{\mathrm{red}}(i)$ is the start of the $i$ th red signal state. In this case, $v_{g}$ can also be determined by

$$
\begin{aligned}
& \text { (i) }\left(v_{k} t_{a}+(1 / 2) a t_{a}^{2}\right)+v_{g}\left(t_{t}-t_{a}\right)=d_{k} \\
& \text { (ii) } t_{a} \leq t_{t} \\
& \text { (iii) } t_{\text {green }}(i) \leq t_{t}<t_{\text {red }}(i) \\
& \text { (iv) } v_{\min } \leq v_{g}=v_{k}+a t_{a} \leq v_{\max } .
\end{aligned}
$$

In constraint (i), $t_{a}$ is the portion of time spent accelerating or decelerating at acceleration or deceleration rate $a$, and $d_{k}$ is the distance between the $k$ th vehicle and the next downstream intersection. Constraints (i) through (iii) ensure that the vehicle will receive a green signal when arriving at the intersection. Constraint (iv) limits the guided velocity to the roadway speed limit.

(2) Acceleration and Deceleration Limit Constraints. The acceleration and deceleration are manipulated to track the target speed as closely as possible while maintaining a safe distance from the front vehicle. The bounds on the acceleration and deceleration are represented by

$$
\begin{aligned}
& 0 \leq a_{\text {acceleration }}^{k} \leq a_{\text {acceleration }}^{\max } \\
& 0 \leq a_{\text {deceleration }}^{k} \leq a_{\text {deceleration }}^{\max }
\end{aligned}
$$

where $a_{\mathrm{acceleration}}^{\max }$ and $a_{\mathrm{deceleration}}^{\max }$ depend on the vehicle type.

(3) The Minimum Safe following Distance Constraint. The minimum safe distance between a vehicle and the preceding vehicle is a function of the vehicle speed and is chosen as [29]

$$
x_{p}-x_{k} \geq \alpha \cdot v_{k}+\beta,
$$

where $\beta$ is a "static gap" parameter that determines the minimum distance needed for the vehicle to stop and $\alpha$ is a "dynamic gap" parameter that indicates the extra time gap given increased speed. When the vehicle is approaching a red light, the light is considered similar to a stopped vehicle, and the position $x_{p}$ is fixed at the light. This ensures that the vehicle will come to a stop at a distance of $\beta$ from the light $\left(x_{p} \geq x_{k}+\beta\right)$.

2.3. Eco-Index Calculation Module. In the eco-index calculation module, the vehicle fuel consumption and $\mathrm{CO}_{2}$ emissions models consider the real-time speed, acceleration, and deceleration. In macroscopic models, the average speed and speed correction factors ( $\mathrm{SCFs}$ ) are used as the basic input 
parameters [30]. However, the SCFs were developed using data collected with fixed driving cycles. Thus, this approach may not adequately capture the effects of driving and vehicle dynamics on fuel consumption and emissions, especially when assessing traffic management strategies [31]. CMEM [32] was developed at a microscopic level to predict secondby-second fuel consumption and emissions using operational activities of a vehicle. Implementing the same basic physical principles as CMEM, the PERE model [33] was designed to generate fuel consumption rates for MOVES, which is the new generation emission model from the United States Environmental Protection Agency, based on the vehicle's on-road load. However, both CMEM and PERE require interfacing with various traffic models. The complexity mainly arises from the following requirements: (1) detailed parameters for vehicle classification, engine, and transmission and (2) application programming interface (API) combined with the speed optimization model for real-time calculation of fuel consumption and emissions. Thus, these models do not apply to fuel consumption impact assessments based on the state of traffic flow.

Studies have shown a corresponding relationship between the fuel consumption per unit distance and vehicle speed and acceleration $[11,31,34]$; however, speed or acceleration alone is inadequate for researching fuel consumption. Thus, there is a need is to discover another parameter associated with both the speed and acceleration to discuss the regularity of fuel consumption.

2.3.1. Vehicle Specific Power (VSP). The normalization parameter used in this study is based on characterizing the VSP of individual vehicles. The VSP has been shown to be a strong descriptor of vehicle fuel consumption and emissions [35] and is defined as the instantaneous tractive power per unit mass of the vehicle. For a typical light-duty vehicle, VSP (in $\mathrm{kW} /$ metric ton) can be calculated as

$$
\mathrm{VSP}=v \cdot(1.1 \cdot a+0.132)+0.000302 \cdot v^{3}
$$

where $v$ is the vehicle speed $(\mathrm{m} / \mathrm{s})$ and $a$ is the acceleration $\left(\mathrm{m} / \mathrm{s}^{2}\right)$. Using (6), we can conclude that when the speed is zero, VSP must be zero and that when acceleration is negative, VSP may be a negative value. Thus, VSP is still a vector although it is being used as a descriptor of the motor vehicle power demand. When a vehicle slows down, the load force acting on the vehicle and traveling direction is inverted, corresponding to a negative value of VSP.

VSP-based approaches have recently been accepted in fuel consumption and emission modeling for two main reasons. First, VSP considers the power needed to overcome aerodynamic drag and rolling resistance and that needed to increase the kinetic and potential energy of a vehicle. Thus, VSP has a direct physical interpretation and strong statistical correlations with fuel consumption and emissions. Second, as shown in (6), by using typical parameters for a certain vehicle type, VSP can be calculated based on vehicle speed and acceleration.

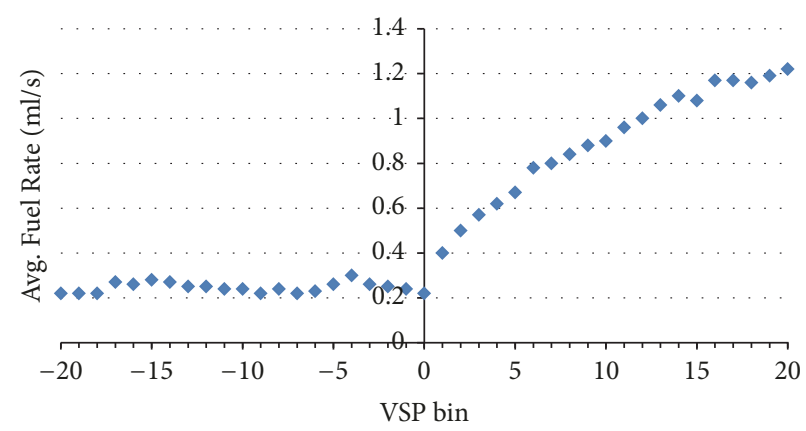

Figure 3: Average fuel rates (mL/s) in each VSP bin for a 2007 model Volkswagen.

2.3.2. Statistical Modeling of Fuel Consumption and VSP. Considerable research has been conducted on modeling the relationship between fuel consumption and VSP [36-38]. This study selected a 2007 Volkswagen model as an example to investigate the relationship between the fuel consumption and VSP distribution of light-duty vehicles.

To analyze the relationship between the VSP and fuel consumption per second more accurately, a binning approach is used. The VSP data are binned into $1 \mathrm{~kW} / \mathrm{t}$ categories, and the average fuel consumption rate and the $95 \%$ confidence interval for each VSP bin are calculated [38]. The results are shown in Figure 3 for the 2007 Volkswagen model. The average fuel rates increase monotonically with VSP for positive bins, while they tend to be relatively lower and roughly constant for negative bins.

(1) Piecewise Modeling of Instantaneous Fuel Consumption and VSP. Statistical analysis found that, in urban transportation, negative VSP bins accounts for approximately $15 \%$ of the fuel consumption. Of the total fuel consumption, $68 \%$ occurs when VSP is positive, and 17\% occurs when the value of VSP is zero. Based on the above analysis, a piecewise model was adopted. The piecewise function of the instantaneous fuel rate (FR, $\mathrm{mL} / \mathrm{s}$ ) versus VSP is shown as follows:

FR

$$
= \begin{cases}0.2508, & \text { for VSP }<-0.5 \\ 0.22, & \text { for }-0.5 \leq \mathrm{VSP}<0.5 \\ 0.0411 \cdot \mathrm{VSP}+0.4629, & \text { for VSP } \geq 0.5 .\end{cases}
$$

In the regression equation, the standard deviation is 0.13 when VSP $<-0.5$, and $R^{2}=0.9618$ when VSP $\geq 0.5$. Thus, the regression results are significant. In addition, the above piecewise function is not continuous. The values of FR in the interval $-0.5 \leq \mathrm{VSP}<0.5$ are lower than those in other ranges, which is caused by the idle speed control of the vehicle.

(2) Fuel Consumption per Unit Distance. Considering that FR is the instantaneous fuel consumption in each time unit (second), we take the integrals of FR and instantaneous velocity in 
this time period to obtain the total fuel consumption and total distance traveled, respectively. The fuel consumption per unit distance (FU) of this light-duty vehicle can then be calculated as follows:

$$
\mathrm{FU}=\frac{\int \mathrm{FR} d t}{\left(\int v d t / d\right)},
$$

where $\mathrm{FU}$ is the fuel consumption per kilometer, $\mathrm{mL} / \mathrm{km} ; v$ is the instantaneous velocity, $\mathrm{m} / \mathrm{s}$; and $d$ is the unit distance, $1,000 \mathrm{~m}$

The speed data obtained from the actual test usually have a unit of meters per second, or even per 0.1 second, and are not continuous variables. Thus, we can use (9) instead of (8).

$$
\mathrm{FU}=\frac{\sum_{i} \mathrm{FR}_{i}}{\left(\sum_{i} v_{i} / d\right)}
$$

From the above analysis, it can be concluded that if continuous speed data are acquired, we can calculate the fuel consumption per unit distance of a specific vehicle. If a vehicle is driving at a constant speed $v\left(v_{\min } \leq v \leq v_{\max }\right),(9)$ can be expressed as

$$
\begin{aligned}
& \mathrm{FU}^{\prime} \\
& =d \\
& \quad \cdot \frac{\left(0.0411 \cdot\left(0.132 \cdot v+0.000302 \cdot v^{3}\right)+0.4629\right)}{v} .
\end{aligned}
$$

Assuming that $d=1,000 \mathrm{~m}$, we can obtain the minimum value of $\mathrm{FU}^{\prime}$. The calculation results indicate that the most fuel-efficient speed for this light-duty vehicle is $95.51 \mathrm{~km} / \mathrm{h}$ and that the minimum fuel consumption (per kilometer) is $31.61 \mathrm{~mL} / \mathrm{km}$. The theoretical calculations of this study and the values based on actual tests match well, demonstrating that the model can represent the actual situation well.

2.3.3. $\mathrm{CO}_{2}$ Emissions Model. Studies have found that, in urban driving, the trends of $\mathrm{CO}_{2}$ emissions and fuel consumption are highly consistent, with correlations of up to 0.99 [38]. According to the mass conservation law, after fuel combustion in the engine, the total carbon mass in the exhaust gas is equal to that in the fuel. Carbon atoms exist in the forms of carbon dioxide $\left(\mathrm{CO}_{2}\right)$, carbon monoxide $(\mathrm{CO})$, and hydrocarbons $(\mathrm{HC})$ in the exhaust gas. The instantaneous fuel consumption is calculated from emissions of $\mathrm{CO}_{2}, \mathrm{CO}$, and $\mathrm{HC}$ using the carbon balance method, as shown in

$$
\begin{aligned}
\mathrm{FR} \cdot \mathrm{SG}= & \left(\mathrm{ER}_{\mathrm{CO}_{2}} \cdot F_{\mathrm{CO}_{2}}+\mathrm{ER}_{\mathrm{CO}} \cdot F_{\mathrm{CO}}+\mathrm{ER}_{\mathrm{HC}} \cdot F_{\mathrm{HC}}\right) \\
& \cdot \frac{1}{g_{\mathrm{C}}},
\end{aligned}
$$

where FR is the instantaneous fuel rate, $\mathrm{mL} / \mathrm{s}$; $\mathrm{SG}$ is the fuel density, $0.749 \mathrm{~g} / \mathrm{mL}$ at $15^{\circ} \mathrm{C} ; \mathrm{ER}_{\mathrm{CO}_{2}}, \mathrm{ER}_{\mathrm{CO}}$, and $\mathrm{ER}_{\mathrm{HC}}$ are the emission rates of $\mathrm{CO}_{2}, \mathrm{CO}$, and $\mathrm{HC}$, respectively, g/s; $\mathrm{F}_{\mathrm{CO}_{2}}$, $F_{\mathrm{CO}}$, and $F_{\mathrm{HC}}$ are the weight fractions of carbon in $\mathrm{CO}_{2}, \mathrm{CO}$, and $\mathrm{HC}$ emissions and are $0.273,0.429$, and 0.866 for gasoline

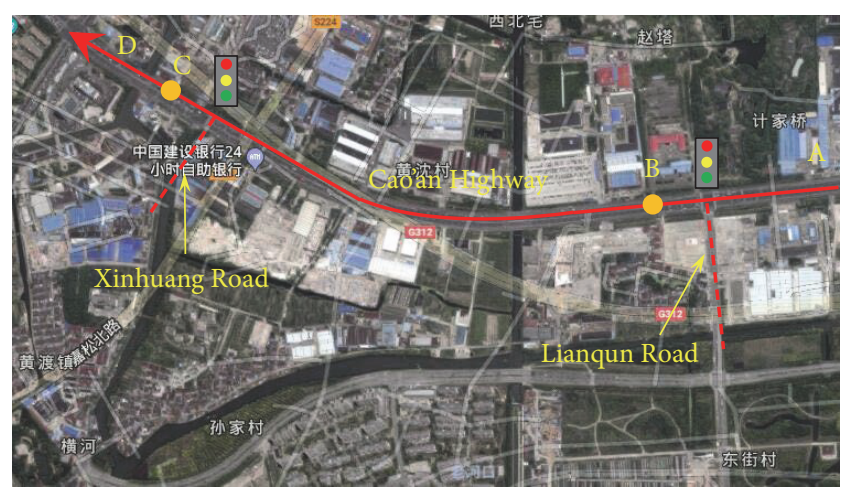

FIgURE 4: Test bed snapshot.

vehicles, respectively; and $g_{C}$ is the carbon percentage of gasoline by weight, which has a typical value of $86.4 \%$.

The proportions of $\mathrm{CO}$ and $\mathrm{HC}$ in all the exhaust gas are less than $2 \%$ and are especially low in strict emission control conditions; thus, they have almost no effect on the balance equation. Thus, the influences of $\mathrm{CO}$ and $\mathrm{HC}$ were neglected in this study when analyzing the relationship between $\mathrm{CO}_{2}$ emissions and fuel consumption. Thus, (11) can be simplified as

$$
\mathrm{ER}_{\mathrm{CO}_{2}}=\mathrm{FR} \cdot 2.370 \text {. }
$$

\section{Case Study}

3.1. Test Bed. A section of Cao'an Highway near the Jiading Campus of Tongji University, Shanghai, China, was selected as the test bed for analysis. The experimental section is $1,700 \mathrm{~m}$ long, with eight lanes in two directions. As illustrated in Figure 4, two adjacent signalized intersections are 1,200 m apart and a curved segment is included between them.

In the experiment, drivers traveled from point $\mathrm{A}$ to point $\mathrm{D}$. To eliminate additional errors, such as starting and stopping and unskilled operation, only the data collected between point $\mathrm{B}$ ( $50 \mathrm{~m}$ from the downstream Lianqun Road) and point $C$ (100 $\mathrm{m}$ from the downstream Xianghuang Road) were taken as valid. In addition, during the experiment the vehicle speed and acceleration were collected every 0.1 seconds.

The other parameters, for example, traffic composition and link capacity, were collected via field surveys. In all the experiments, the vehicle mass is assumed to be $m=1365 \mathrm{~kg}$. $v_{\max }$ is set to $80 \mathrm{~km} / \mathrm{h}$ according to the road speed limit, and $v_{\min }$ is set to $40 \mathrm{~km} / \mathrm{h}$ considering that a driver is likely to deviate from the desired speed if the guided velocity is lower than $40 \mathrm{~km} / \mathrm{h}$. The maximum acceleration and maximum deceleration are assumed to be $a_{\text {acceleration }}^{\max }=3.5 \mathrm{~m} / \mathrm{s}^{2}$ and $a_{\text {deceleration }}^{\max }=-7.0 \mathrm{~m} / \mathrm{s}^{2}$, respectively. In most cases, the vehicles travel at a constant feasible deceleration, called desired deceleration, which is assumed to be $a_{\text {deceleration }}^{\text {deire }}=$ $-3.0 \mathrm{~m} / \mathrm{s}^{2}$.

3.2. Experiments. The dynamic eco-driving speed guidance system consists of the drivers, vehicles, driving environment, 


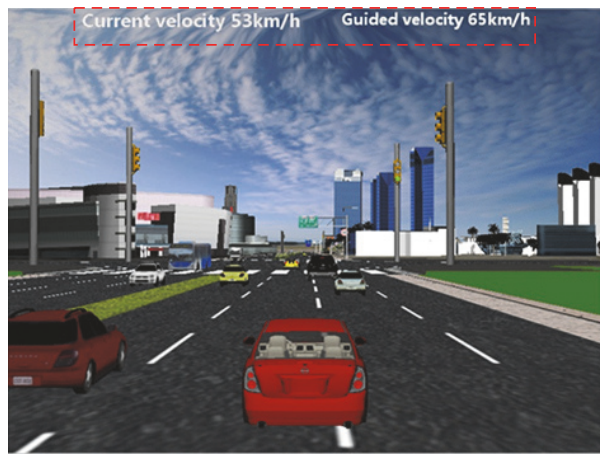

(a) Scene screenshot

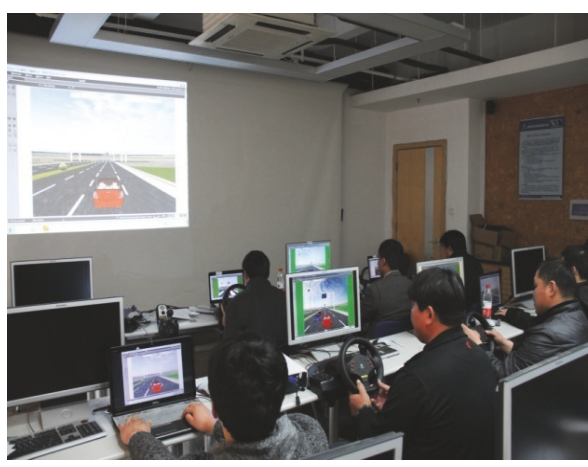

(b) Multivehicle driving experiment

Figure 5: Multivehicle driving simulator based experiments.

and speed guidance strategy. Experiments were conducted using driving simulators because we investigated a nonexistent situation and the experiment could create potentially dangerous situations due to unknown effects. The driving simulator experiment can also describe the influence of driver's psychology and behavior stimulated by the dynamic speed information. In this study, the program-oriented multidriving simulator was chosen as the experimental platform. Each computer was equipped with a special steering wheel, gear, pedals, and other hardware devices. 3DS Max and VIRTOOLS modeling software were used to build the virtual driving scene, as shown in Figure 5(a). The DESGS was programmed using the script language provided by VIRTOOLS. Using an intranet environment, the vehicles could be driven and interact in the same experimental setup, as shown in Figure 5(b).

Fifteen typical drivers from Tongji University and the nearby community were recruited to participate in the experiments. The ages of the participants were between 21 and 50 and followed a normal distribution. All the participants had held their driving licenses for more than five years and drove more than 8,000 kilometers annually.

The participants completed the driving experiments in two scenarios: vehicles with and without eco-speed guidance. Each scenario was simulated 24 times. The data, including time, vehicle position coordinates $(x, y)$, current velocity, and guided velocity, were collected.

\section{Results of Analysis}

The 3rd vehicle of the group was placed in one of two scenarios: vehicles without speed guidance and vehicles with eco-speed guidance. An example of an eco-driving vehicle velocity profile compared to a non-eco-driving vehicle velocity profile is shown in Figure 6. The eco-driving vehicle had a much smoother velocity trajectory, yielding lower fuel consumption, as shown in Figure 7. The velocity profile shows that the vehicle never stopped with hard braking at red lights. With predictive use of signal information, the eco-driving vehicle scheduled its velocity based on the guided velocity, thus passing through intersections without coming to a stop.

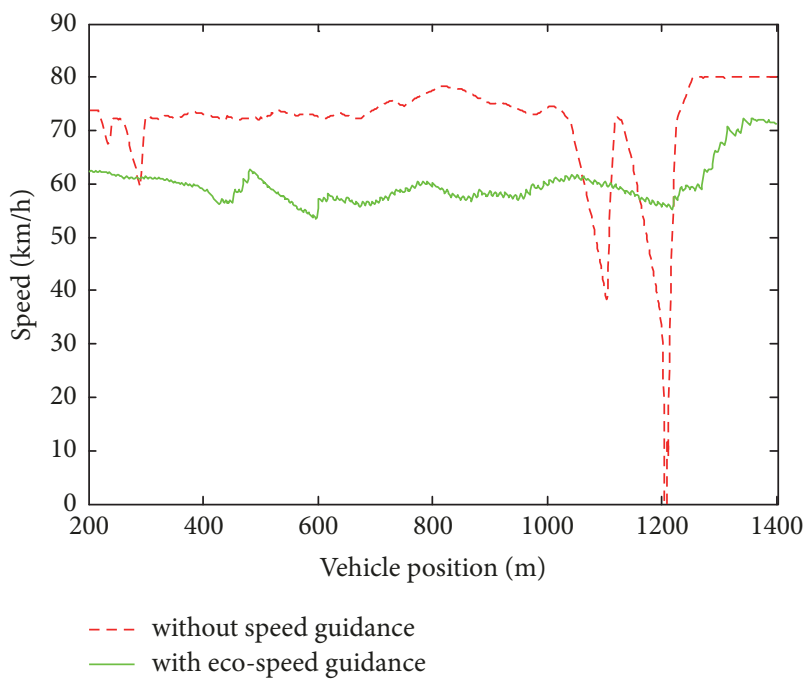

FIGURE 6: Velocity trajectory comparisons with and without speed guidance.

Figure 7 illustrates the instantaneous fuel rate comparison of two vehicles in different scenarios. Without speed guidance, the vehicles on the segment were driven randomly but usually had to stop at the downstream intersection. Frequent acceleration or deceleration required much fuel. The fuel rate curve was observed to be flat with speed guidance, increasing only when the speed advice was updated.

For comparison, the travel time, number of stops, fuel consumption, and $\mathrm{CO}_{2}$ emissions were selected as the evaluation indexes. Figure 8 compares the results for the vehicles with and without eco-speed guidance. The results for both cases are given in terms of the average value of all the velocity profiles in the scenarios. According to Figure 8, for the vehicles with eco-speed guidance, the number of stops was significantly reduced; the total fuel consumption decreased from $53.01 \mathrm{~mL}$ to $39.88 \mathrm{~mL}$ (approximately 25\% lower); and the $\mathrm{CO}_{2}$ emissions decreased from $125.71 \mathrm{~g}$ to $94.56 \mathrm{~g}$ (approximately $25 \%$ lower). The average travel time was approximately $4 \%$ shorter for the vehicles with eco-speed 


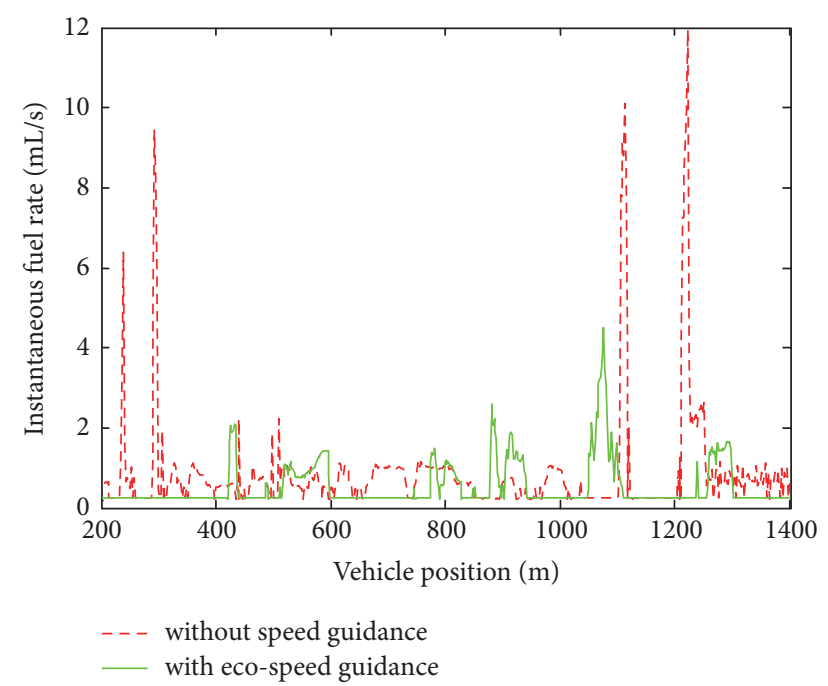

FIGURE 7: Instantaneous fuel rate comparison for vehicles with and without speed guidance.

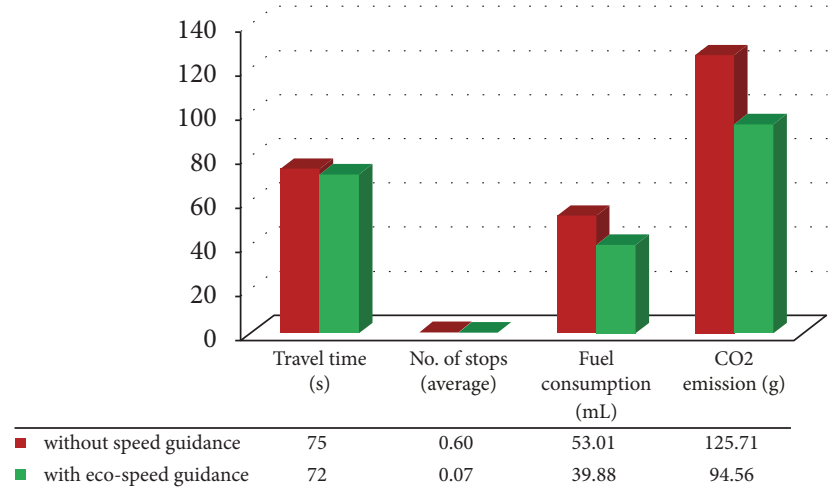

FIGURE 8: Comparison of evaluation indexes for the vehicles with and without speed guidance.

guidance compared to the vehicles without speed guidance, but this difference is not significant. The energy savings and decrease in emissions are remarkable.

\section{Conclusions and Future Work}

To reduce fuel consumption and $\mathrm{CO}_{2}$ emissions for traveling along signalized arterials, a dynamic eco-driving speed guidance strategy (DESGS) was established in this study using real-time signal timing and vehicle positioning information in a CV environment. The developed algorithms help optimize the fuel/emission speed profiles for vehicles approaching signalized intersections. To evaluate the effectiveness of the overall strategy, 15 typical drivers were recruited to participate in interactive experiments using multivehicle driving simulators. The main conclusions obtained in this study are summarized as follows:

(i) The optimization-based rolling horizon and DP approach can dynamically adjust the speed advice according to the vehicle's spatial-temporal trajectories. The eco-driving vehicles had much smoother velocity variations than the unguided vehicles.

(ii) The results given by the VSP-based fuel consumption model demonstrated good agreement with the field consumption curve. Thus, the VSP model was integrated into the optimal eco-driving speed calculation model.

(iii) The DESGS showed remarkable energy savings and decreased emissions. Both fuel consumption and $\mathrm{CO}_{2}$ emissions were reduced by approximately $25 \%$ compared with the unguided scenarios.

(iv) The DESGS also showed a significant decrease in the number of stops and a small decrease in the travel time compared to the unguided scenarios.

There are still some ongoing efforts for the study. First, for simplicity, this study only investigated vehicles' fuel/emission speed profiles in a complete CV environment. That is, all the vehicles are CVs which are able to exchange traffic information and communicate with traffic signal controllers to obtain signal information. While in a partial CV environment, only limited vehicles can receive $\mathrm{V} 2 \mathrm{~V}$ and V2I information and a key impact factor on DESGS would be market penetration rate ranging from 0 to $100 \%$. It is supposed that different market penetration rates of CVs would result in different savings of fuel consumption and $\mathrm{CO}_{2}$ emissions through implementing DESGS. Besides, the robustness of the strategy to errors in wireless communication and driver responses should also be analyzed. Next, other impact factors, for example, the length of controlled segment, traffic demands (corresponding to undersaturated or oversaturated conditions), and signal phasing and timing, are supposed to be included in a more comprehensive analysis of the strategy performance. Furthermore, the developed DESGS can be extended to multiple signalized intersections along the arterial. Note that, in the case of multilane approaches, the potential impact of lane changing behavior in practice needs to be carefully considered. These will be our future work.

\section{Conflicts of Interest}

The authors declare that there are no conflicts of interest regarding the publication of this paper.

\section{Acknowledgments}

The authors appreciate the National Natural Science Foundation of China (nos. U1564212, 51508014, and 51422812), the Shanghai Science and Technology Project of International Cooperation (16510711400), and the Fundamental Research Funds for the Central Universities for their support of this research. Thanks also are due to Mr. Dening Niu and Mr. Shenyang Chen for comments and suggestions on an earlier version of this paper submitted to the 92nd Annual Meeting of the Transportation Research Board for presentation only. 


\section{References}

[1] International Energy Agency, "Energy efficiency policy recommendations," in Support of the G8 Plan of Action, IEA, Paris, France, 2008.

[2] M. Barth and K. Boriboonsomsin, "Energy and emissions impacts of a freeway-based dynamic eco-driving system," Transportation Research Part D: Transport and Environment, vol. 14, no. 6, pp. 400-410, 2009.

[3] N. Jollands, P. Waide, M. Ellis et al., "The 25 IEA energy efficiency policy recommendations to the G8 Gleneagles Plan of Action," Energy Policy, vol. 38, no. 11, pp. 6409-6418, 2010.

[4] J. N. Barkenbus, "Eco-driving: an overlooked climate change initiative," Energy Policy, vol. 38, no. 2, pp. 762-769, 2010.

[5] K. Brookhuis and D. De Waard, "Limiting speed, towards an intelligent speed adapter (ISA)," Transportation Research Part F: Traffic Psychology and Behaviour, vol. 2, no. 2, pp. 81-90, 1999.

[6] K. Boriboonsomsin, O. Servin, and M. Barth, Selection of Control Speeds in Dynamic Intelligent Speed Adaptation System: A Preliminary Analysis, EScholarship, University of California, Calif, USA, 2008.

[7] H. Johansson, P. Gustafsson, M. Henke, and M. Rosengren, "Impact of eco driving on emissions. transport and air pollution," in Proceedings of the 12th Symposium, Avignon, France, 2003.

[8] J. Barbé and G. Boy, "On-board system design to optimize energy management," in Proceedings of the European Annual Conference on Human Decision-Making and Manual Control (EAM'06), Valenciennes, France, 2006.

[9] O. H. Koskinen, "Improving vehicle fuel economy and reducing emissions by driving technique," in Proceedings of the 15th ITS World Congress, New York, NY, USA, 2008.

[10] H. Yang, H. Rakha, and M. V. Ala, "Eco-Cooperative Adaptive Cruise Control at Signalized Intersections Considering Queue Effects," IEEE Transactions on Intelligent Transportation Systems, vol. 18, no. 6, pp. 1575-1585, 2017.

[11] K. Ahn, H. Rakha, A. Trani, and M. van Aerde, "Estimating vehicle fuel consumption and emissions based on instantaneous speed and acceleration levels," Journal of Transportation Engineering, vol. 128, no. 2, pp. 182-190, 2002.

[12] Z. Zhang, Y. Wang, P. Chen, Z. He, and G. Yu, "Probe data-driven travel time forecasting for urban expressways by matching similar spatiotemporal traffic patterns," Transportation Research Part C: Emerging Technologies, vol. 85, pp. 476493, 2017.

[13] M. Chen, G. Yu, P. Chen, and Y. Wang, "A copula-based approach for estimating the travel time reliability of urban arterial," Transportation Research Part C: Emerging Technologies, vol. 82, pp. 1-23, 2017.

[14] G. S. Pierre and J. Ehrlich, "Impact of intelligent speed adaptation systems on fuel consumption and driver behavior," in Proceedings of the 15th ITS World Congress, New York, NY, USA, 2008.

[15] M. Li, K. Boriboonsomsin, G. Wu, W. B. Zhang, and M. Barth, "Traffic energy and emission reductions at signalized intersections: a study of the benefits of advanced driver information," International Journal of Intelligent Transportation Systems Research, vol. 7, no. 1, pp. 49-58, 2009.

[16] P. Chen, K. Yin, and J. Sun, "Application of finite mixture of regression model with varying mixing probabilities to estimation of urban arterial travel times," Transportation Research Record, vol. 2442, pp. 96-105, 2014.
[17] P. Chen, G. Yu, X. Wu, Y. Ren, and Y. Li, "Estimation of redlight running frequency using high-resolution traffic and signal data," Accident Analysis \& Prevention, vol. 102, pp. 235-247, 2017.

[18] Research and Innovative Technology Administration. Connected Vehicle Research, http://www.its.dot.gov/connected_vehicle/connected_vehicle.htm, 2012.

[19] Z. Papp, "Situational awareness in intelligent vehicles," in Handbook of Intelligent Vehicles, A. Eskandarian, Ed., pp. 62-78, Springer, 2012.

[20] S. Mandava, K. Boriboonsomsin, and M. Barth, "Arterial velocity planning based on traffic signal information under light traffic conditions," in Proceedings of the 12th International IEEE Conference on Intelligent Transportation Systems, St. Louis, Mo, USA, 2009.

[21] B. Asadi and A. Vahidi, "Predictive cruise control: utilizing upcoming traffic signal information for improving fuel economy and reducing trip time," IEEE Transactions on Control Systems Technology, vol. 19, no. 3, pp. 707-714, 2011.

[22] M. Barth, S. Mandava, K. Boriboonsomsin, and H. Xia, "Dynamic ECO-driving for arterial corridors", in Proceedings of the IEEE Forum on Integrated and Sustainable Transportation System (FISTS '11), pp. 182-188, IEEE, Vienna, Austria, July 2011.

[23] H. Rakha and R. K. Kamalanathsharma, "Eco-driving at signalized intersections using V2I communication," in Proceedings of the 14th IEEE International Intelligent Transportation Systems Conference (ITSC '11), pp. 341-346, IEEE, Washington, DC, USA, October 2011.

[24] H. Bock and K. Plitt, "A multiple shooting algorithm for direct solution of optimal control problems," in Proceedings of the 9th IFAC World Congress, Pergamon Press, Budapest, Romania, 1984.

[25] D. Kraft, "On converting optimal control problems into nonlinear programming problems," in Computational Mathematical Programming, K. Schittkowski, Ed., vol. 15, pp. 261-280, NATO ASI, 1985.

[26] A. E. Bryson and Y. C. Ho, Applied Optimal Control, Taylor \& Francis, New York, NY, USA, 1975.

[27] A. Fröberg and L. Nielsen, "Optimal fuel and gear ratio control for heavy trucks with piece wise affine engine characteristics," in Proceedings of the 5th IFAC Symposium on Advances in Automotive Control, Monterey Coast, Calif, USA, 2007.

[28] B. Saerens, H. Rakha, and E. V. D. Bulck, "Assessment of eco-cruise control calculation methods," in Proceedings of the 91st Annual Meeting of the Transportation Research Board, Washington, DC, USA, 2012.

[29] A. Vahidi and A. Eskandarian, "Research advances in intelligent collision avoidance and adaptive cruise control," IEEE Transactions on Intelligent Transportation Systems, vol. 4, no. 3, pp. 132153, 2003.

[30] C. Brzezinski and P. Enns, "Final facility specific speed correction factors," Printed on Recycled Paper EPA-420-R-01-060, EPA, Washington, DC, USA, 2001.

[31] A. Cappiello, I. Chabini, E. Nam, A. Lue, and M. Abou Zeid, "A statistical model of vehicle emissions and fuel consumption," in Proceedings of the IEEE 5th International Conference on Intelligent Transportation Systems., pp. 801-809, Singapore.

[32] G. Scora and M. Barth, User's Guide: Comprehensive Modal Emissions Mode (CMEM), University of California, Riverside, Calif, USA, 3.01 edition, 2006.

[33] E. K. Nam, "Fuel consumption modeling of conventional and advanced technology vehicles in the physical emission 
rate estimator (PERE)," U.S. Environmental Protection Agency EPA420-P-05-001, EPA.

[34] R. Joumard, A. J. Hickman, and J. Nemerlin, "Modelling of emissions and consumption in urban areas (MODEM)," INRETS, LEN 9213, Bron, France, 1992.

[35] J. L. Jiménez-Palacios, Understanding and Quantifying Motor Vehicle Emissions with Vehicle Specific Power and TILDAS Remote Sensing [Doctoral, thesis], Massachusetts Institute of Technology, Cambridge, Mass, USA, 1999.

[36] H. Wang, L. Fu, Y. Zhou, and H. Li, "Modelling of the fuel consumption for passenger cars regarding driving characteristics," Transportation Research Part D: Transport and Environment, vol. 13, no. 7, pp. 479-482, 2008.

[37] G. Song and L. Yu, "Estimation of fuel efficiency of road traffic by a characterization of VSP and speed based on FCD," in Proceedings of the 88th Annual Meeting of the Transportation Research Board, Washington, DC, USA, 2009.

[38] G. Song and L. Yu, "A practical model approach for evaluation of fuel efficiency for road traffic," in Proceedings of the 87th Annual Meeting of the Transportation Research Board, Washington, DC, USA, 2008 


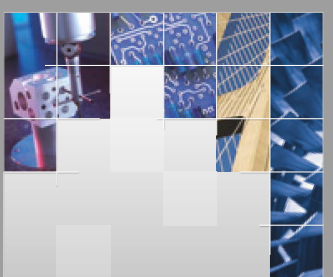

\section{Enfincering}
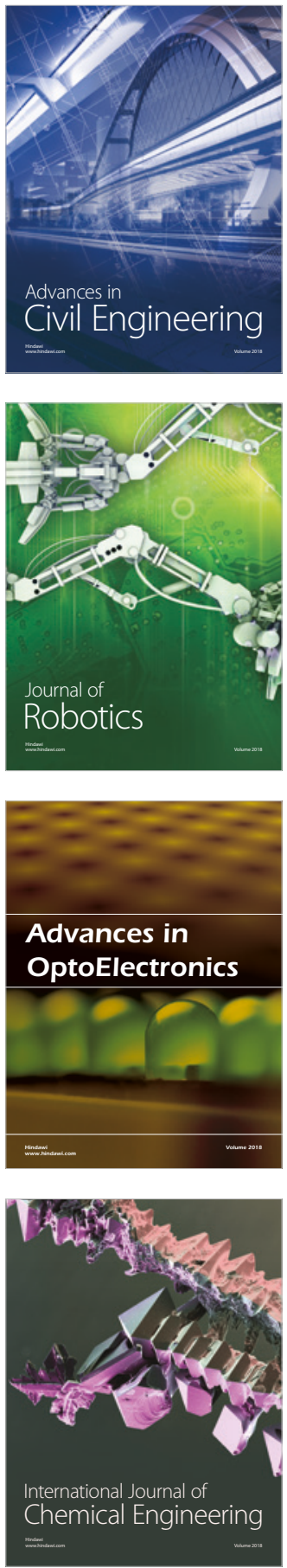

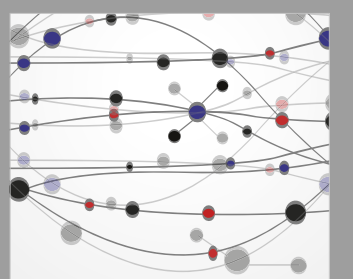

\section{Rotating \\ Machinery}

The Scientific World Journal

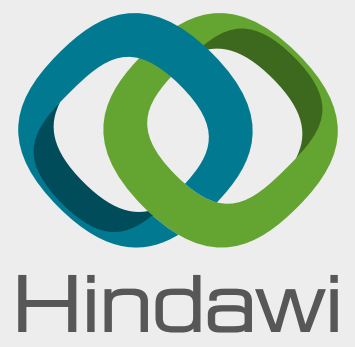

Submit your manuscripts at

www.hindawi.com
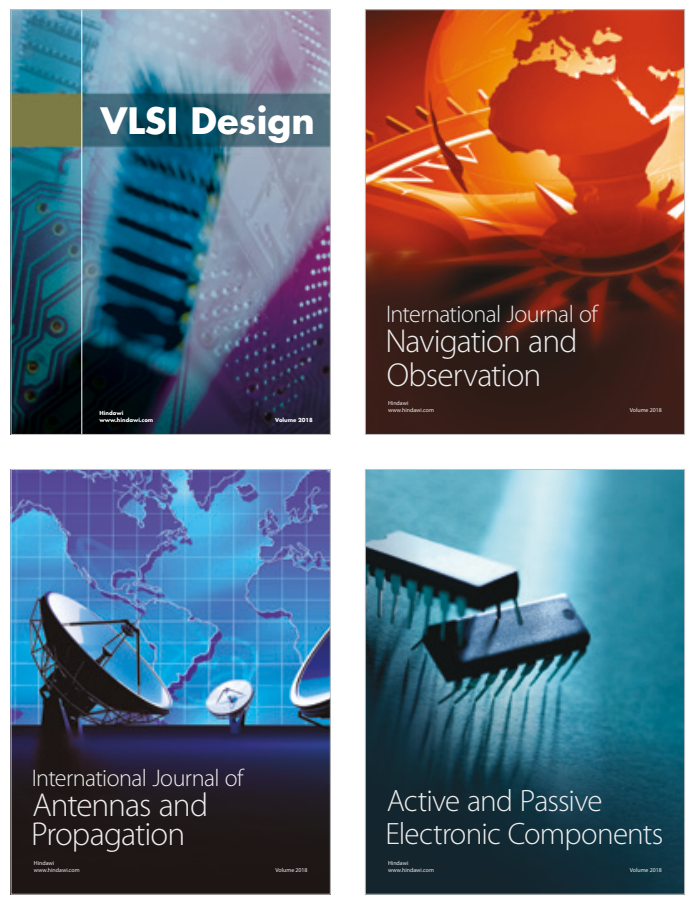
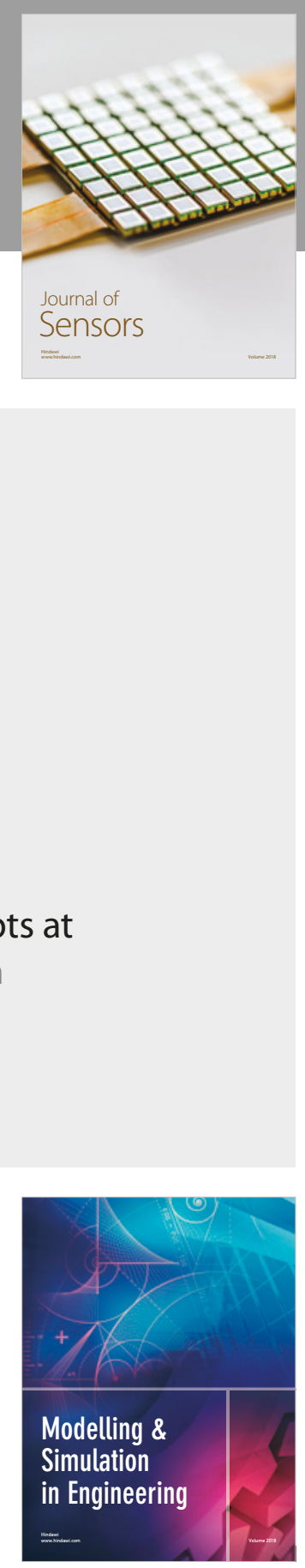

\section{Advances \\ Multimedia}
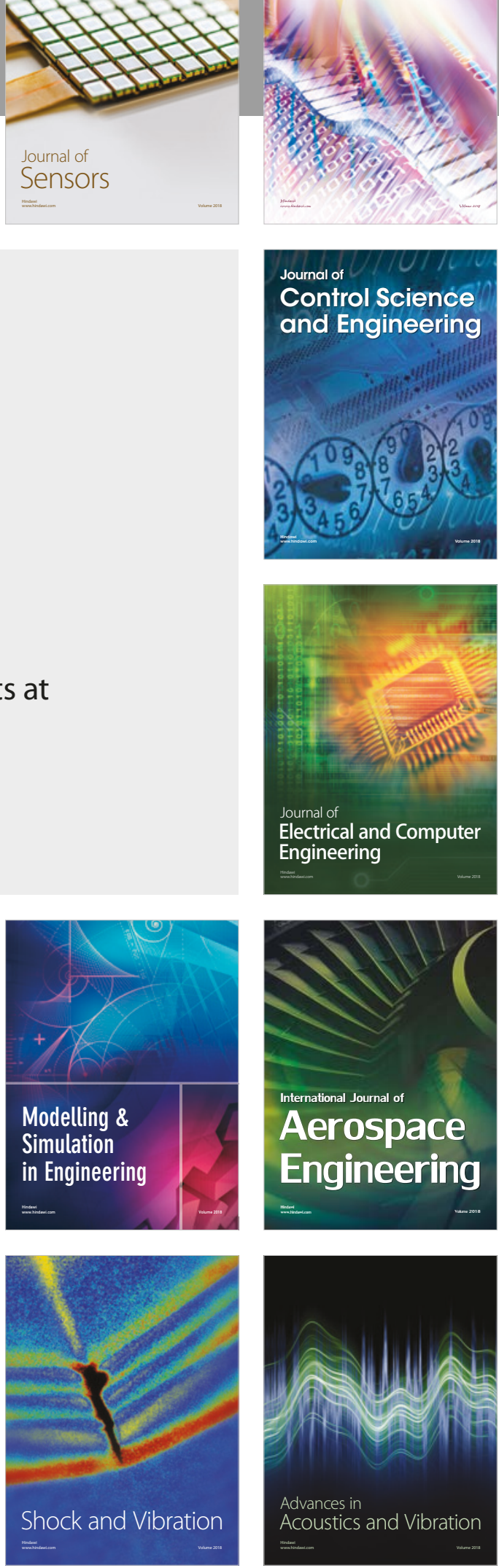